\title{
Modalites D'acquisition Des Terres Et Conflits Fonciers Dans La Sous-Prefecture De Sinfra (Cote d'Ivoire)
}

\author{
Prof. Bakayoko Ismaila, Maître de conférences \\ Dr. Kana Jean Noel Pacôme, Sociocriminologue \\ Dr. Traore Fatoumata, Sociocriminologue \\ Université Félix Houphouët Boigny d'Abidjan, Cote d'Ivoire
}

Doi:10.19044/esj.2020.v16n20p112 URL:http://dx.doi.org/10.19044/esj.2020.v16n20p112

\section{Résumé}

Cette étude vise à expliquer comment à partir des transactions foncières rurales entre populations sédentaires, naissent les conflits fonciers à Sinfra (Côte d'Ivoire). Elle s'appuie sur la théorie de l'acteur de Blumer qui privilégie l'approche idiographique dans l'explication des phénomènes sociaux. L'enquête a été effectuée auprès d'une population de 112 individus repartis selon les catégories suivantes $\mathrm{N} \mathrm{1:}$ autorités coutumières et administratives et $\mathrm{N} 2$ : ruraux et cultivateurs dans les 16 villages que compte cette sous-préfecture. En termes de techniques de recherche, l'étude a eu recours à la documentation, l'observation et l'entretien. Les données révèlent que la plupart des litiges fonciers observés à Sinfra résultent des modalités d'acquisition des terres et des rixes liées aux contrats de cession élaborés par des acteurs ruraux sans qualification qui, tout en n'associant pas des acteurs habilités en la matière, se heurtent à des difficultés syntaxiques et à la reproduction textuelle de leurs aspirations dans le cadre des contrats. Parallèlement, ces partenaires fonciers qui croient que leurs aspirations sont toutes définies dans le texte, évoquent des droits qui n'apparaissent pas clairement dans le rédigé et de ce fait, agissent non pas selon le contrat écrit mais selon leurs aspirations, générant ainsi entre eux, heurts et rixes. Pour circonscrire ces conflits, l'étude plaide en faveur de l'intégration des acteurs administratifs dans le processus d'acquisition des terres rurales.

Mots-clés: Modalité, Acquisition, Terre, Conflit 


\title{
Terms of Acquisition of Land and Land Conflicts in the Sub-Prefecture of Sinfra (Cote d'Ivoire)
}

\author{
Prof. Bakayoko Ismaila, Maître de conférences \\ Dr. Kana Jean Noel Pacôme, Sociocriminologue \\ Dr. Traore Fatoumata, Sociocriminologue \\ Université Félix Houphouët Boigny d'Abidjan, Cote d'Ivoire
}

\begin{abstract}
This study aims to explain how, from rural land transactions between sedentary populations, land conflicts arise in Sinfra (Côte d'Ivoire). It is based on the theory of the actor of Blumer who privileges the idiographic approach in the explanation of social phenomena. The survey was carried out on a population of 112 individuals divided into the following categories $\mathrm{L} 1$ : customary and administrative authorities and L 2: rural people and farmers in the 16 villages of this sub-prefecture. In terms of research techniques, the study used documentation, observation and interview. The data reveal that most of the land disputes observed in Sinfra result from the terms of land acquisition and brawls linked to the sale contracts drawn up by unskilled rural actors who, while not associating actors authorized in the matter, encounter syntactic difficulties and the textual reproduction of their aspirations within the framework of contracts. At the same time, these land partners who believe that their aspirations are all defined in the text, evoke rights that do not appear clearly in the wording and therefore act not according to the written contract but according to their aspirations, thus generating between them, clashes and brawls. To reduce these conflicts, the study calls for the integration of administrative actors in the process of acquiring rural land.
\end{abstract}

Keywords: Modality, Acquisition, Land, Conflict

\section{Introduction}

La littérature relative aux modalités d'acquisition des terres en milieu rural est prolixe. La plupart des recherches se sont articulées autour de la prédominance du tutorat dans le tissu rural africain (Chauveau, Colin, Jacob, Lavigne et Le Meur, 2006 ; Le Roy, 1996), de la présentation des droits contenus dans l'usage de la terre concédée (Kouamé, 2010 ; Lavigne, 2002). En d'autres termes, de l'espace en tant que tel et de sa texture organique et minérale contenues dans la nomenclature «faisceaux de droits » (Ouedraogo, 
2009) et de la discrimination foncière de la femme dans l'échiquier familial (Berriane, 2016 ; Fatiha, 2011 ; Doka et Monimart, 2004 ; Sohinto, 2001). Les modalités d'acquisition des terres dans le terroir africain, restent focalisées sur des pratiques traditionnelles locales (Agnissan, 2010 ; Faye, 2008) dans un contexte social précaire et négocié entre ces populations au profil sociodémographique distinct (Yoda, 2009). Ainsi, des modalités d'acquisition de terres, le tutorat apparait comme un système d'intégration microsociale de l'allogène (Lawali, Mormont et Yamba, 2014 ; Bontemps, 2006 ; Chauveau, 2004 ; Gnabeli, 2008) qui reste contraint de se conformer aux rituels d'établissement des liens avec son hôte (Nakabanda, 2017 ; Thareau, 2011).

Depuis quelques années, relativement aux antagonismes fonciers observés dans la plupart des contrées villageoises (Ratiaray, 1989), un certain nombre de publications scientifiques suggèrent l'intégration incrémentale des normes juridiques dans le processus d'acquisition des terres (Oumarou, 2008 ; Mumbere et Rignall, 2017). En d'autres termes, il s'agit du jumelage des normes traditionnelles et juridiques dans le processus d'attribution des terres en milieu rural (Bobo, 2012; Tsongo et Kitakya, 2006). Par ailleurs, la question de l'accès à la terre et la sécurisation foncière constitue une préoccupation fondamentale aussi bien pour les Etats africains que pour les populations rurales (Houdeingar, 2009; Bologo, 2004). La pression démographique et la saturation foncière constatées dans les différentes régions des pays ouest-africains (Kakule, 2010 ; Tallet et Pare, 1999), ont conduit ces Etats à réinterpréter le processus de négociation des droits fonciers en milieu rural (Coulibaly, 2015 ; Kouamé, 2010). Le système des droits fonciers de ces zones forestières fortement peuplées, résultait d'une politique privilégiant la mise en valeur de la terre conformément à la coutume (Bonnecase, 2001 ; Chauveau, 2000 ; Ibo, 2006 ; Merabet, 2006). Dans le contexte rural ivoirien, la terre est à la fois sacralisée (Meillassoux, 1964) humanisée, socialisée (Kana, 2017) et n'est pas un objet comme les autres, susceptible d'aliénation (Agnissan, 2010). Ainsi, si pour obtenir une portion de terre en milieu rural, le demandeur reste soumis à une démarche villageoise clivée et centrée sur des compensations financières ou matérielles et sur l'implication de divinités ancestrales (Zongo, 2009), cela reste plus affirmé dans le centre-ouest ivoirien (Meillassoux, 1964 ; Kana, 2017 ; Bonnecase, 2001), zone à prédominance forestière.

Ces contributions scientifiques, bien qu'elles permettent d'avoir une vue d'ensemble sur le processus traditionnel d'acquisition des terres dans la majorité des contrées régionales et sous-régionales africaines, occultent cependant la dynamique du lien entre modalités d'acquisition des terres et conflit fonciers. Or, il nous semble évident que c'est à travers le processus d'attribution des terres et des conventions résultantes que naissent la plupart des conflits fonciers dans ces contrées locales. C'est cette question qui sera au 
centre de nos préoccupations dans la présente communication. Il s'agit pour nous, d'analyser et d'expliquer comment, à partir des transactions foncières rurales entre populations sédentaires, naissent les conflits fonciers à Sinfra.

La théorie utilisée dans le cadre de cette étude, est celle de l'acteur de Blumer (1969). Blumer postule en effet que pour comprendre le comportement humain, il faut recourir non pas à la signification des choses dans leur valeur intrinsèque mais plutôt la signification des choses selon les acteurs sociaux. Dès los, on ne pourra véritablement comprendre la question des conflits inhérents aux modalités de transmission des terres à Sinfra, que par la recherche d'une part de la signification de la terre pour ces populations sédentaires et d'autre part, par la compréhension du faussé social entre les aspirations des partenaires fonciers et le contenu de ces transactions.

L'analyse d'un tel sujet suscite l'interrogation suivante : Quel est le lien entre les modalités d'acquisition des terres et les conflits fonciers dans la sous-préfecture de Sinfra?

L'hypothèse qui sous-tend un tel énoncé se formule de la manière suivante : Les conflits fonciers à Sinfra naissent et émergent du processus d'attribution des terres et des conventions orales ou écrites qui en résultent.

\section{Méthodologie \\ Site et participants}

Le cadre choisi pour conduire cette recherche est la sous-préfecture de Sinfra. Notre connaissance du terrain d'étude, des principes culturels existants, de la fréquence des contradictions foncières, des acteurs ruraux et administratifs ont milité en faveur du choix de ce terrain d'étude. Notons également que la sous-préfecture de Sinfra s'étend sur une superficie d'environ $1612 \mathrm{~km}^{2}$ et est limitée dans la partie Nord par le département de Bouaflé, au sud par les départements d'Oumé et de Gagnoa, à l'est par le district de Yamoussoukro et à l'ouest par le département d'Issia (BNETD, 2005). Elle a une population estimée à environ 90.711 habitants (RGPH, 2014). Celle-ci est majoritairement jeune et hétérogène constituée d'autochtones «kwênins », d'allochtones et des ressortissants des pays de l'Afrique de l'ouest.

L'enquête a été menée auprès d'un échantillon de 112 individus repartis dans les 16 villages de la sous-préfecture de Sinfra pour à la fois diversifier les sources d'information et pour répondre à un souci d'efficacité, de rapidité et de réduction des coûts de l'enquête. Pour les besoins de la recherche, la technique d'échantillonnage non probabiliste a été privilégiée puisqu'elle consiste à recruter des participants de façon non aléatoire (Daddi, 2010) et permet par ricochet d'atteindre une représentativité impartiale de la population à l'étude pour que toute estimation basée sur l'échantillon soit représentative de la population mère (Ardilly, 1994). Le choix s'est donc porté 
sur les techniques par choix accidentel (ruraux, cultivateurs) et par choix raisonné (autorités coutumières et administratives).

L'échantillon a été regroupé en deux catégories sociales :

- Catégorie N1 : autorités coutumières et administratives

- Catégorie N2 : ruraux et cultivateurs

Pour préserver l'anonymat des enquêtés dans la transcription des verbatim, les lettres initiales des noms ont été retenues.

\section{Instruments de recueil des données}

Trois instruments ont été exploités : la documentation, l'observation et les différents entretiens (entretien individuel et focus group) pour recueillir les données du terrain. Dans la documentation, nous avons effectué une recension des contributions antérieures à l'effet d'avoir une vue générale et claire de notre objet d'étude. Au niveau de l'observation, nous avons à la fois observé et participé au processus d'attribution de terres dans quelques villages (Béliata, Digliblanfla, Blontifla et Kouêtinfla). Enfin, des entretiens individuels et focus group effectués auprès des nouveaux acquéreurs de terres et des membres des familles kwênins ont permis d'obtenir des données relatives aux modalités d'acquisition des terres à Sinfra et des joutes $\mathrm{y}$ afférentes.

\section{Méthodes d'analyse des données}

L'étude s'est appuyée sur deux méthodes d'analyse des données : analyses quantitative et qualitative. L'analyse quantitative a permis de regrouper les données (issues des questions fermées), de dresser des tableaux de distribution statistique en vue de leur interprétation en termes statistiques. L'analyse qualitative a privilégié la démarche culturaliste pour fonder toute dynamique d'interprétation scientifique sur le système culturel des kwênins, leur conception de la terre et le contexte social des transactions foncières. L'usage des questions ouvertes a permis de recenser des verbatim axés sur le vécu des acteurs, leur ressenti afin d'en faire une analyse.

\section{Résultats}

Les résultats s'articulent autour de deux axes : modalités d'acquisition des terres et les conflits inhérents à ces modalités d'acquisition des terres.

\subsection{Modalités d'acquisition des terres}

\subsubsection{Typologie des modalités d'acquisition des terres}

\begin{tabular}{|l|l|l|l|l|l|l|}
\cline { 2 - 7 } \multicolumn{1}{c|}{} & Héritage & Achat & Mise en gage & Prêt & Métayage & Total \\
\hline Effectifs & 47 & 30 & 06 & 08 & 21 & 112 \\
\hline Fréquence & $41,96 \%$ & $26,78 \%$ & $5,35 \%$ & $7,14 \%$ & $18,75 \%$ & $100 \%$ \\
\hline
\end{tabular}


A l'analyse, l'héritage $(47: 41,96 \%)$ est plus utilisé que l'achat $(26,78 \%)$, la mise en gage $(5,35 \%)$, le prêt $(7,14 \%)$ et le métayage $(18,75 \%)$ dans la transmission de terres à Sinfra. Cette prédominance de l'héritage dans la consolidation foncière est liée au fait que dans la coutume Gouro, les terres ont été reparties selon les familles, lignages et tribus par les ancêtres. Chaque village ou lignage était affecté sur des terres sectorielles de sorte à éviter les effets de dispersion des membres, de désordre ou de litiges entre autochtones eux-mêmes. C'est pourquoi dans la sous-préfecture de Sinfra, l'appellation d'un village donne implicitement des informations sur la situation géographique et les limites des différentes portions de terres appartenant aux habitants de cette lignée autochtone. A titre illustratif, nous pouvons mentionner que dans le village Digliblanfla, selon les répartitions des terres par les ancêtres, les autochtones disposent des forêts «plaplowouo", «voêagloudji », «valsigoêwi », «zablagoli », « goazi », « gloutaplô » que chaque autochtone est censé connaitre (noms, emplacements et limites) de sorte à mieux les transmettre des descendants aux descendants.

L'héritage est donc le moyen de transmission le plus utilisé par la population rurale. Les différents prêts, mises en gage et ventes que l'on observait depuis quelques temps à Sinfra, ont progressivement été substitués par le métayage qui semble être au confluent de ces deux types de transactions. Cette pratique relativement nouvelle connait un grand succès dans la souspréfecture puisque par son truchement, les autochtones peuvent valoriser leur portion de terre sans toutefois s'investir eux-mêmes dans les activités champêtres.

\subsubsection{Héritage}

"Il se fonde sur la conception originale suivant laquelle, la terre a un caractère essentiellement familial, lignager. Ainsi, à la mort d'un parent, ses terres sont partagées entre ses frères et ses fils; les filles en sont exclues car elles seront appelées à quitter le domicile familial en vue d'un éventuel mariage » (D. cultivateur à Proniani). Autrement, l'héritage est un moyen de transmission foncière à caractère exclusivement utérin avec une exclusion de la descendance féminine en raison de la probabilité d'un mariage avenir. Mais dans certains cas, lorsque le défunt n'a pas de descendants masculins, les sages du village statuent et envisagent la possibilité d'attribuer à titre exceptionnel des terres à ses filles qui désirent les cultiver.

Par ailleurs, selon ce même enquêté, "la société kwênin de Sinfra est régie par le patriarcat». La parenté s'établit donc en ligne masculine d'où la prédominance de la descendance paternelle. Ainsi, les frères et les descendants directs du défunt sont privilégiés dans l'attribution et le partage des biens en général et des terres en particulier. C'est seulement, lorsque ceux-ci font défaut que les biens reviennent aux collaborateurs, aux cousins du défunt et à leur 
descendance. Mais dans tous les cas, les parents de l'épouse et les gendres sont exclus et le partage est fonction de certains critères qualitatifs tels que l'âge, l'influence familiale et lignagère, le sens de la responsabilité et l'engagement dans les activités champêtres. Le partage des richesses est fonction de ces critères à moins que le défunt ait, de façon confidentielle ou par testament, laissé des instructions différentes.

\subsubsection{Achat}

C'est une transaction foncière qui s'établit entre un propriétaire terrien qui manifeste un besoin financier et un allochtone qui manifeste un besoin foncier. Cette transaction est rédigée par ces acteurs ou un tiers sollicité pour la circonstance puis entérinée par les parties en présence et ci-possible des témoins.

\subsubsection{Mise en gage}

Elle est définie par l'enquêté G. (Cultivateur à Manoufla) comme «un contrat par lequel un propriétaire remet sa terre à un créancier et lui donne le droit de garder et d'exploiter cette terre jusqu'au remboursement de sa dette ». En d'autres termes, la mise en gage est un mode de consolidation foncière qui accorde une jouissance totale au nouvel acquéreur durant la durée du contrat et ne prescrit aucune forme de reconnaissance ou de civilités du créancier à l'égard du propriétaire et vis-versa. Toutefois, celui-ci reste exclusivement tenu d'effectuer des cultures vivrières sur la portion de terre en raison de l'incertitude de la date de remboursement.

\subsubsection{Prêt}

Le prêt est un système transactionnel par lequel le propriétaire d'une terre met à la disposition d'un tiers, une partie ou la totalité de sa propriété pour en tirer profit avant que le besoin ne s'impose à lui. Ainsi, pour Z. (Chef du village, Tricata) «le bénéficiaire de ce droit de gestion, exerce comme le propriétaire de la terre, les mêmes fonctions d'occupation, d'exercice, de gestion notamment sur la portion de terre qui lui a été attribuée. Ce droit de propriété qu'exerce le bénéficiaire est différent du droit de propriété exclusif en ce sens qu'il est tenu de rendre compte de sa gestion au propriétaire. A travers une convention de prêt, le bénéficiaire jouit des bénéfices de la ressource foncière qu'il a sollicitée et obtenue auprès de son propriétaire légitime, mais cette jouissance implique en contrepartie, le respect de certaines clauses auprès desquelles, il a obtenu le droit d'exercice ». Ces clauses peuvent être sociales ou foncières.

$\mathrm{Au}$ niveau des clauses sociales : elles sont de nature relationnelle et prescrivent très peu de lignes de conduite que le bénéficiaire devra avoir envers son «têrêzan » et octroient plutôt une primauté au respect des valeurs 
et normes culturelles. Cette pratique dite ancestrale par certains enquêtés s'observe dans les villages assez reculés de la ville (Dégbesséré, Koizra, Proziblanfla), presque coupées des nouvelles réalités capitalistes du marché actuel, où les populations jusque-là sont restées fidèles aux pratiques culturelles ancestrales.

Concernant les clauses foncières : elles sont de nature prescriptive. C'est un système qui met l'emphase sur la contrepartie financière ou matérielle équivalente au don. Dans cette forme de transaction, le «têrêzan » attend du bénéficiaire, des gestes en nature de façon régulière (trimestriellement ou annuellement) selon les termes définis dans le cadre du contrat foncier.

\subsubsection{Métayage ou contrat «zépa »}

Cette pratique assez fréquente dans la sous-préfecture de Sinfra, est perçue par M. (Chef du service cadastre de la direction départementale de l'agriculture) comme « un contrat entre un propriétaire terrien et un migrant, spécialisé dans les activités champêtres, qui consiste pour le propriétaire à céder une partie ou la totalité de son espace foncier à ce migrant qui, devra dans une période déterminée, valoriser l'espace de sorte à en faire un champ productif; une sorte de planter-partager. Cette durée de mise en valeur qui vacille régulièrement entre cinq et huit ans, est repartie de la façon suivante : $2 / 3$ pour le propriétaire terrien et $1 / 3$ pour le métayeur.

3.1.2 Fréquence des modalités d'acquisition des terres par village

\begin{tabular}{|c|c|c|c|c|c|c|c|c|c|c|c|}
\hline Modes & \multicolumn{2}{|c|}{ Héritage } & \multicolumn{2}{|c|}{ Achat } & \multicolumn{2}{|c|}{ Mise en gage } & \multicolumn{2}{|l|}{ Prêt } & \multicolumn{2}{|c|}{ Métayage } & Total \\
\hline Blontifla & 04 & $40 \%$ & 03 & $30 \%$ & 01 & $10 \%$ & 00 & $00 \%$ & 02 & $20 \%$ & $10 \quad 8,82 \%$ \\
\hline Douafla & 05 & $41,6 \%$ & 04 & $33,3 \%$ & 00 & $00 \%$ & 01 & $8,3 \%$ & 02 & $16,7 \%$ & $\begin{array}{ll}12 & 10,71 \%\end{array}$ \\
\hline Proniani & 06 & $46,2 \%$ & 03 & $23 \%$ & 01 & $7,6 \%$ & 00 & $00 \%$ & 03 & $21,1 \%$ & $1311,60 \%$ \\
\hline Benhuafla & 01 & $33,3 \%$ & 00 & $00 \%$ & 00 & $00 \%$ & 01 & $33,3 \%$ & 01 & $33,3 \%$ & $03 \quad 2,67 \%$ \\
\hline Béliata & 04 & $44,4 \%$ & 03 & $33,3 \%$ & 00 & $00 \%$ & 01 & $11,2 \%$ & 01 & $11,2 \%$ & $098,03 \%$ \\
\hline Digliblanfla & 06 & $42,9 \%$ & 05 & $35,8 \%$ & 01 & $7,1 \%$ & 00 & $00 \%$ & 02 & $14,2 \%$ & $12,50 \%$ \\
\hline Bègoneta & 04 & $36,3 \%$ & 03 & $27,2 \%$ & 00 & $00 \%$ & 03 & $27,2 \%$ & 01 & $9,1 \%$ & $9,82 \%$ \\
\hline Kouétinfla & 06 & $40 \%$ & 04 & $26,7 \%$ & 02 & $13,3 \%$ & 00 & $00 \%$ & 03 & $20 \%$ & $13,39 \%$ \\
\hline Manoufla & 01 & $25 \%$ & 00 & $00 \%$ & 00 & $00 \%$ & 01 & $25 \%$ & 02 & $50 \%$ & $3,57 \%$ \\
\hline Barata & 01 & $33,3 \%$ & 00 & $00 \%$ & 00 & $00 \%$ & 01 & $33,3 \%$ & 01 & $33,3 \%$ & $03 \quad 2,67 \%$ \\
\hline Tricata & 01 & $33,3 \%$ & 01 & $33,3 \%$ & 00 & $00 \%$ & 00 & $00 \%$ & 01 & $33,3 \%$ & $03 \quad 2,67 \%$ \\
\hline Bérita & 01 & $100 \%$ & 00 & $00 \%$ & 00 & $00 \%$ & 00 & $00 \%$ & 00 & $00 \%$ & $0,89 \%$ \\
\hline Dégbesséré & 01 & $50 \%$ & 00 & $00 \%$ & 00 & $00 \%$ & 00 & $00 \%$ & 01 & $50 \%$ & $1,78 \%$ \\
\hline Proziblanfla & 01 & $50 \%$ & 01 & $50 \%$ & 00 & $00 \%$ & 00 & $00 \%$ & 00 & $00 \%$ & $1,78 \%$ \\
\hline Koizra & 01 & $33,3 \%$ & 01 & $33,3 \%$ & 00 & $00 \%$ & 00 & $00 \%$ & 01 & $33,3 \%$ & $2,67 \%$ \\
\hline Koblata & 04 & $57,1 \%$ & 02 & $28,2 \%$ & 01 & $14,28 \%$ & 0 & $00 \%$ & 00 & $00 \%$ & $6,25 \%$ \\
\hline Total & 47 & $41,96 \%$ & 30 & $26,78 \%$ & 06 & $5,35 \%$ & 08 & $7,14 \%$ & 21 & $18,75 \%$ & $112100 \%$ \\
\hline
\end{tabular}


Dans les villages Proniani (06: 46,2\%), Kouêtinfla (06: 40\%) et Douafla (05: 41,6\%), l'héritage est affirmé (47: 41,96\%). Dans les autres villages, les fréquences restent moyennes et varient $(33,3 \%$ à $44,4 \%)$. L'achat est privilégié à Digliblanfla $(05: 35,8 \%)$, Douafla $(04: 33,3 \%)$ et Kouêtinfla $(04: 26,7 \%)$. Le prêt est fortement perçu à Bégonéta $(03: 27,2 \%)$. Le métayage est relativement présent dans l'ensemble des villages avec des fréquences variantes $(16,7 \%$ à $33,3 \%)$. Cette dominance des différentes modalités d'acquisitions des terres dans les villages Proniani, Kouêtinfla, Douafla, Digliblanfla et Bégonéta est lié au fait que ces villages sont recentrés dans le centre-ville de Sinfra. Avec la saturation foncière actuelle, les terres locales sont sujettes à diverses méthodes de consolidation: parenté (héritage), proposition financière (achat), amicale (prêt), protectionniste (mise en gage, métayage).

\subsection{Conflits fonciers liés aux transactions foncières 3.2.1 Typologie des conflits fonciers}

\begin{tabular}{|c|l|c|c|}
\cline { 2 - 4 } \multicolumn{1}{c|}{ Conflits fonciers } & \multicolumn{1}{|c|}{ Effectifs } & $\%$ \\
\hline \multirow{4}{*}{ Typologie } & P1 : Conflit autour de la gestion de l'héritage & 57 & $50,89 \%$ \\
\cline { 2 - 4 } & P2 : Conflits liés à l'imprécision des clauses d'achat et de prêt & 11 & $9,82 \%$ \\
\cline { 2 - 4 } & P3 : Litiges liés au remboursement pour la mise en gage & 06 & $5,35 \%$ \\
\cline { 2 - 4 } & P4 : Rixe inhérente à la nature du contrat Zépa & 38 & $33,92 \%$ \\
\hline \multirow{2}{*}{} & Total & 112 & $100 \%$ \\
\cline { 2 - 4 }
\end{tabular}

A l'analyse du tableau, quatre principaux types de conflits (conflit autour de la gestion de l'héritage, conflits liés à l'imprécision des clauses d'achat et de prêt, litiges liés au remboursement pour la mise en gage et rixe inhérente à la nature du Zépa et non-respect de la période de récolte) sont identifiés dans la sous-préfecture de Sinfra. Le paramètre $« P 1$ : Conflit autour de la gestion de l'héritage » (57:50,89\%) est plus affirmé que les paramètres «P2: Conflits liés à l'imprécision des clauses d'achat et de prêt » (11: $9,82 \%), \ll P 3$ : Litiges liés au remboursement pour la mise en gage » et «P4 : Rixe inhérente à la nature du Zépa et non-respect de la période de récolte » $(38: 33,92 \%)$.

Quelques verbatim attestent ces données :

$\mathrm{Au}$ niveau du conflit autour de la gestion de l'héritage familial, l'enquêté D. (planteur à Blontifla) affirme que «quand le papa, avant de mourir n'a pas prévu un héritier pour gérer les terres, il y a des palabres sur celui qui va gérer sa ». Il ressort de ces propos qu'en milieu familial, lorsque le père donateur des terres n'a pas, par testament ou instruction, désigné un héritier avant sa mort, les fils utérins s'engagent dans des joutes individuelles 
pour la gestion des terres héritées. Toutefois, même si dans la plupart des cas, c'est l'ainé qui est plus habilité à hériter en raison du droit d'ainesse, l'hégémonie de celui reste régulièrement remise en cause dans la structure familiale où chacun prétend avoir les compétences requises pour gérer ces terres. Ces informations sont confirmées par les propos de S. (coiffeur à Bégonéta), pour qui «les jeunes de Bégonéta veulent aujourd'hui qu'on ne donne pas les terres à quelqu'un qui peut les vendre demain pour partir en ville». Ainsi, des facteurs subjectifs tels que l'instabilité psychologique de l'ainé ou son goût à l'aventure sont quelques fois prétextés par l'ensemble des fils du défunt, pour réclamer par divers moyens (verbal, physique et relationnel) la gestion de ces biens familiaux. Par ailleurs, si la majorité des membres de la famille se dotent de divers moyens pour s'accaparer la gestion des biens familiaux, l'objectif ne parait pas nécessairement la volonté d'accroître les avoirs de la famille (développement de diverses espèces culturales), mais plutôt de bénéficier des avantages pluriels liés à cette gestion et de pouvoir contracter des prêts sous le couvert des portions de terres familiales. Mais au-delà de ce fait, Z. (cultivateur à Tricata) affirme que "lorsque le papa distribue les terres à ses enfants avant de mourir, d'autres n'acceptent pas le partage et attendent la mort du vieux pour chercher à prendre la terre des autres ». En d'autres termes, même si le père donateur laisse un testament ou procède personnellement à la distribution des terres à ses descendants, ce partage ne fait souvent pas l'unanimité et constitue quelques fois la niche de conflits à venir. Ainsi, les «frustrés » de cette distribution attendent selon l'enquêté, la mort du père pour entreprendre des campagnes à la fois pacifiques et violentes d'appropriation des espaces de leurs frères. Et dans ce contexte, il apparait que les plus faibles (physiquement et mystiquement) paient le plus lourd tribut dans cette appropriation de masse, des espaces des uns par les autres à l'intérieur de la sphère familiale ou lignagère de la sous-préfecture de Sinfra.

Concernant les conflits liés à l'imprécision des clauses d'achat et de prêt, l'enquêté V. (archiviste de la Sous-préfecture de Sinfra) mentionne que "pour vendre des terres, le vendeur et le payeur utilisent des petits papiers souvent sales et mal entretenus pour écrire dessus et choisissent dans le tas, des témoins à qui, ils vont remettre un peu d'argent pour signer leur contrat ». Dès lors, il ressort que ni le vendeur, ni l'acheteur ne se soucie de l'élaboration d'un contrat de vente ou de prêt en bonne et due forme, mais s'en tiennent au simple fait que l'un a reçu l'argent et l'autre, la terre. Ainsi, avec ce type de contrat élaboré quelque fois dans la précipitation avec un évitement des acteurs locaux habilités en matière d'élaboration des contrats fonciers, ces acteurs se retrouvent quelques temps plus tard, opposés sur certains points de droit concernant ce contrat élaboré par des individus au niveau scolaire basique. En outre, il est important de remarquer que les acteurs ruraux font montre de 
difficultés syntaxiques criantes et de ce fait, ne mentionnent pas quelques informations fondamentales des contrats. A ce niveau, les propos de F. (planteur à Kouetinfla) sont poignants : "souvent ceux qui font leur contrat disent beaucoup de choses qu'ils n'écrivent pas ». Dès lors, il parait difficile à moins d'investigations approfondies, de redéfinir le contrat sur la base de leurs propos souvent contradictoires avec des témoins déserteurs après réception de leurs commissions. C'est pourquoi, le collectif des chefs traditionnels de Sinfra a érigé un chapelet de conditions visant à intégrer la chefferie traditionnelle dans le processus de cession foncière afin de suivre et entériner la transaction avant toute entreprise de bornage traditionnel des parcelles consolidées. Le porte-parole du collectif des chefs de villages affirme à ce propos que «les chefs de village ont entrepris de vastes campagnes d'information et de sensibilisation sur l'interdiction formelle d'élaborer des transactions sournoises, sous peine d'annulation de contrat et de ses effets ».

$\mathrm{Au}$ niveau des litiges liés au remboursement dans la mise en gage, l'enquêté Z. (planteur à Kouêtinfla) pense que la mise en gage est d'abord une "garantie pour que celui qui emprunte l'argent ait la pression pour rembourser ». En d'autres termes, la mise en gage suppose d'une part un créancier qui prête de l'argent à un planteur et d'autre part, que ce planteur ait mis en gage une portion de terre ou une terre déjà cultivée pour donner l'assurance au créancier d'un remboursement certain. Ainsi, lorsque le calendrier de remboursement établi dans le cadre de ce contrat n'est pas respecté, ces acteurs se voient se menacer, s'affronter clandestinement dans les plantations avant de remonter à l'échelle villageoise pour intenter une gestion coutumière. Par ailleurs, si l'enquêté M. (Chef du service cadastre de la direction départementale de l'agriculture) affirme que «pour les cas de mise en gage, nous avons du mal à intervenir », cela s'explique par le fait que la majorité de ces contrats se font de gré à gré en dehors de tout contexte légal et les accusations des uns envers les autres, restent fortuites poussant ces belligérants à esquisser d'autres solutions qu'ils trouvent quelques fois dans la violence physique ou mystique.

En ce qui concerne, les rixes inhérentes à la nature du contrat «zépa», Mr Z. (chef de la tribu Sian) note une double séries de points litigieux : " $a u$ niveau du contenu du contrat et au niveau de la période de récolte convenue dans le contrat de métayage ». Dans le premier cas (contenu du contrat de cession foncière), propriétaires terriens et métayeurs trouvent fréquemment des points de désaccord. Il se pose en effet la question de savoir si ce métayage s'applique exclusivement sur la récolte ou à la fois sur la récolte et la terre. Ce faisant, ces acteurs sollicitent fréquemment l'intervention de la chefferie traditionnelle qui, elle-même, à moins d'un délaissement, rencontre d'énormes difficultés dans la gestion de cette question. Mais au-delà de cette rixe 
spécifique à la nature du contrat "zépa », se pose quelques fois des difficultés liées au non-respect de la période de récolte en raison des maladies fréquentes du verger. L'approche culturale à Sinfra étant principalement archaïque, les métayeurs ont du mal à protéger le verger contre certaines maladies dont la plus redoutable reste «le swoollen shoot ». Cette maladie saisonnière, décime fréquemment les plants de cacaoyers à Sinfra. Toute chose qui n'est pas de nature à faciliter les activités du métayeur dont le délai de production qui constitue la quintessence du contrat, est temporellement circonscrit (enquêté de la direction départementale de l'agriculture et certains ruraux). Dès lors, les désaccords entre propriétaires terriens et métayeurs sont inévitables parce que pour la plupart des tèrèzan interrogés, le contrat ne peut plus avoir de sens à partir du moment où la période de récolte n'a pas été respecté par le métayeur. Ces divergences au départ verbales, se métamorphosent assez rapidement en violences physiques avec sollicitation d'acteurs extérieurs puisque tandis que le métayeur négocie un report de la date de récolte en raison des maladies du verger, les tèrèzan tentent de remettre en cause le contrat de cession et de réquisitionner l'espace litigieux. De ce type spécifique de conflit qui voit s'entrecroiser différents intérêts et acteurs sociaux, les chefs traditionnels naviguent entre chercher à concilier les protagonistes tout en conservant les intérêts fonciers de chacun ou agir pour éviter les risques de dégénérescence du désaccord entre particuliers en un conflit intra ou intercommunautaire.

\subsubsection{Occurrence des paramètres de conflits par rapport aux villages de} la sous-préfecture de Sinfra

\begin{tabular}{|c|rc|lc|cc|cc|rr|}
\hline $\begin{array}{c}\text { Paramètres } \\
\text { Villages }\end{array}$ & \multicolumn{2}{|c|}{ P1 } & \multicolumn{2}{|c|}{ P2 } & \multicolumn{2}{|c|}{ P3 } & \multicolumn{2}{|c|}{ P4 } & \multicolumn{2}{c|}{ Total } \\
\hline Blontifla & 04 & $57,1 \%$ & 01 & $14,2 \%$ & 00 & $00 \%$ & 02 & $28,6 \%$ & 07 & $6,25 \%$ \\
\hline Douafla & 05 & $50 \%$ & 00 & $00 \%$ & 01 & $10 \%$ & 04 & $40 \%$ & 10 & $9,92 \%$ \\
\hline Proniani & 05 & $41,6 \%$ & 01 & $8,3 \%$ & 01 & $8,3 \%$ & 05 & $41,6 \%$ & 12 & $10,71 \%$ \\
\hline Benhuafla & 01 & $50 \%$ & 00 & $00 \%$ & 00 & $00 \%$ & 01 & $50 \%$ & 02 & $1,78 \%$ \\
\hline Béliata & 04 & $40 \%$ & 01 & $10 \%$ & 01 & $10 \%$ & 04 & $40 \%$ & 10 & $9,92 \%$ \\
\hline Digliblanfla & 05 & $41,6 \%$ & 01 & $8,3 \%$ & 01 & $8,3 \%$ & 05 & $41,6 \%$ & 12 & $10,71 \%$ \\
\hline Bègoneta & 04 & $40 \%$ & 01 & $10 \%$ & 01 & $10 \%$ & 04 & $40 \%$ & 10 & $9,92 \%$ \\
\hline Kouétinfla & 06 & $37,5 \%$ & 03 & $18,8 \%$ & 01 & $6,2 \%$ & 06 & $37,5 \%$ & 16 & $14,28 \%$ \\
\hline Manoufla & 02 & $50 \%$ & 01 & $25 \%$ & 00 & $00 \%$ & 01 & $25 \%$ & 04 & $3,57 \%$ \\
\hline Barata & 02 & $50 \%$ & 00 & $00 \%$ & 00 & $00 \%$ & 02 & $50 \%$ & 04 & $3,57 \%$ \\
\hline Tricata & 03 & $75 \%$ & 00 & $00 \%$ & 00 & $00 \%$ & 01 & $25 \%$ & 04 & $3,57 \%$ \\
\hline Bérita & 04 & $80 \%$ & 00 & $00 \%$ & 00 & $00 \%$ & 10 & $20 \%$ & 05 & $4,46 \%$ \\
\hline Dégbesséré & 01 & $50 \%$ & 01 & $50 \%$ & 00 & $00 \%$ & 00 & $00 \%$ & 02 & $1,78 \%$ \\
\hline Proziblanfla & 03 & $75 \%$ & 00 & $00 \%$ & 00 & $00 \%$ & 01 & $25 \%$ & 04 & $3,57 \%$ \\
\hline Koizra & 03 & $100 \%$ & 00 & $00 \%$ & 00 & $00 \%$ & 00 & $00 \%$ & 03 & $2,67 \%$ \\
\hline Koblata & 05 & $71,4 \%$ & 01 & $14,2 \%$ & 00 & $00 \%$ & 01 & $14,2 \%$ & 07 & $6,25 \%$ \\
\hline Total & 57 & $50,89 \%$ & 11 & $9,82 \%$ & 06 & $5,35 \%$ & 38 & $33,92 \%$ & 112 & $100 \%$ \\
\hline
\end{tabular}


Dans les villages Koblata (71,4\%), Benhuafla (50\%), Kouêtinfla (37,5\%), Proniani (41,6\%), Douafla (50\%) et Digliblanfla (41,6\%), les conflits portant sur la gestion de l'héritage $(50,89 \%)$ sont plus affirmés. Cela s'explique par la corruption passive de quelques héritiers ou ayants droits de terres, qui ayant conscience de l'interdiction de ventes clandestines de terres, s'y prêtent en charriant l'ensemble des membres de la famille dans des joutes intrafamiliales à l'effet de revendiquer et consolider les espaces aux allogènes (demandeurs de terre). Les fréquences importantes de conflits liés à la nature du contrat Zépa (Béliata : 40\% ; Bégonéta : 40\% ; Digliblanfla : 41,6\% ; Kouêtinfla : 37,5\%) s'expliquent par le fait que la jeunesse rurale de Sinfra devient de plus en plus aventurière et nourrit fréquemment des ambitions d'emplois faciles et bien rémunérés dans la capitale économique et de ce fait, ils y accourent en grand nombre. Relativement, L. (métayeur à Kouêtinfla) soutient que «les jeunes cultivateurs de Sinfra fuient les travaux champêtres pour aller chercher bon travail à Abidjan. Et puis, ils donnent leurs terres en zépa ». Les villages recentrés autour du centre-ville (Béliata, Bégonéta, Digliblanfla et Kouêtinfla) paient le plus lourd de cette désertion rurale et des contrats de «zépa » effectués à la hâte par ces aventuriers.

\section{Discussion et Conclusion}

L'examen des résultats de l'étude montrent que l'objectif et l'hypothèse ont été validés. La plupart des litiges fonciers observés à Sinfra en effet, résultent des modalités d'acquisitions des terres et des conflits inhérents aux contrats élaborés dans le cadre de ces transactions. Par ailleurs, même si ces contrats s'établissent sur des papiers avec des témoins, ceux-ci ne s'inscrivent pas dans un contexte légal qui préconise l'entérinement de ces transactions par des acteurs habilités à cet effet dans la sous-préfecture de Sinfra. La majorité de ces contrats bien qu'écrits, restent mal définis sur des petits papiers par ces acteurs majoritairement analphabètes, qui tout en n'associant pas les acteurs habilités en matière d'élaboration de contrat, se heurtent à des difficultés syntaxiques et à la reproduction textuelle de leurs aspirations dans le cadre du contrat. Parallèlement, ces partenaires fonciers qui croient que leurs aspirations sont toutes définies dans le texte, évoquent des droits qui n'apparaissent pas clairement dans le rédigé et de ce fait, agissent non pas selon le contrat écrit mais selon leurs aspirations, générant ainsi entre eux, heurts et rixes.

Ces résultats valident les travaux qui plaident en faveur du jumelage des normes traditionnelles et juridiques dans le processus d'attribution des terres rurales (Chauveau, 2004; Houdeingar, 2009; Oumarou, 2008 ; Mumbere et Rignall, 2017). Pour ces auteurs, l'intégration progressive de normes juridiques au processus coutumier d'acquisition des terres rurales (Zongo, 2009) permet de matérialiser de façon textuelle les aspirations des 
partenaires fonciers (Chauveau, 2004 ; Houdeingar, 2009) et par ricochet, de réduire les conflits fonciers (Mumbere et Rignall, 2017). Mais, au-delà de l'agrégation des normes culturelles et administratives, il serait nécessaire dans le cadre de l'élaboration des contrats fonciers, d'intégrer les acteurs institutionnels (chefs de villages, sous-préfet, préfet, acteurs de la direction départementale de l'agriculture) dans le cadre des transactions. Ceux-ci devront, en dehors de l'anticipation et la gestion des litiges fonciers, participer au processus rédactionnel des contrats fonciers et au suivi des interactions entre ces partenaires fonciers en vue de prévenir d'éventuels litiges.

Toutefois, s'il est vrai que de nombreux conflits fonciers à Sinfra trouvent leur explication dans la genèse du processus d'acquisition des terres, il n'en demeure pas moins que tous les conflits fonciers observés dans cette localité n'émanent pas tous du processus d'attribution des terres. Des facteurs endogènes (mauvaise gestion de l'héritage foncier familial, discrimination foncière de minorités sédentaires) et exogènes (effet de l'urbanisation et consolidation de terres, implication d'acteurs extra-ruraux dans le jeu foncier) aux acteurs sont susceptibles de constituer des combustibles au métamorphisme de divergences foncières en conflits entre populations sédentaires. Dès lors, ne serait-il pas judicieux d'envisager des pistes de réflexion sur ces aspects à l'effet d'avoir une vue claire sur les facteurs endogènes et exogènes qui sont à l'origine de la relation foncière dualiste d'une part entre les ayants droits et d'autre part, entre les populations sédentaires dans le centre-ouest ivoirien et particulièrement à Sinfra?

\section{References :}

1. AGNISSAN, A. (2010). Dynamique urbaine et conservation des forêts sacrées : enjeux théoriques et paradoxes épistémologiques. Colloque international BiodiverCities.

2. ARDILLY, P. (1994). Les techniques de sondage. Paris: Ed TECHNIP

3. BERRIANE, Y et RIGNALL, K. (2017). La fabrique de la coutume au Maroc: le droit des femmes aux terres collectives. Paris : L'Harmattan.

4. BLUMER, H. (1969). Interactionnisme symbolique: Perspective et méthode. Paris : PUF.

5. BOBO, S. (2012). Enjeux fonciers et dynamiques des rapports sociaux en milieu rural ouest-africain. Sénégal : Université Cheikh Anta Diop -UMR GRED.

6. BOLOGO, E. (2004). Transferts intergénérationnels de la terre et mutations foncières dans l'Ouest du Burkina Faso. Paris : Le Journal des Sciences Sociales. 
7. BONNECASE, V. (2001). Les étrangers et la terre en Côte d'Ivoire à l'époque coloniale. Montpellier : Cedex.

8. BONTEMPS, B. (2006). Modes d'accès à la terre et fondement de l'ordre social. Le tutorat en question sur le terroir villageois de DéguéDégué (Burkina Faso). Montpellier: "Les frontières de la question foncière"

9. BNETD (2005). Plan stratégique de développement du département de Sinfra: Diagnostic provisoire : Rapport provisoire, circulation restreinte.

10. CHAUVEAU, J.P. (2004). Conflit, crise de la ruralité et crise foncière en Côte d'Ivoire forestière : le « tutorat » entre autochtones et migrants au centre des tensions. Atelier CLAIMS : Mali.

11. CHAUVEAU, J-P., COLIN, J-P., JACOB, J-P., LAVIGNE, D-P et LE MEUR, P. (2006). Modes d'accès à la terre, marchés fonciers, gouvernance et politiques foncières en Afrique de l'ouest : résultats du projet de recherche claims. Londres : IIED

12. COULIBALY, G. (2015). Système matrilinéaire, conflits fonciers intrafamiliaux et mutations sociales chez les Agni sanwi de côte d'Ivoire: anthropologie des recompositions en cours. France : European Scientific Journal.

13. DADDI, H. (2010). Analyse du comportement du consommateur dans le marché algérien des assurances. Mémoire Ingénieur d'Etat en statistique appliquée. ENSSEA (EX INPS).

14. DOKA, M et MONIMART, M. (2004). Pression foncière et nouvelles normes d'accès à la terre : Vers une déféminisation de l'agriculture au Sud Niger? Londres : IIED

15. FATIHA, D. (2011). Droits fonciers des femmes au Maroc Entre complexité du système foncier et discrimination. Etude sur le terrain des droits fonciers des femmes au Maroc. Maroc : Les Etudes et Essais du Centre Jacques Berque.

16. FAYE, J. (2008). Genre et foncier : une équation non encore résolue au Sénégal. Paris : Karthala

17. GNABELI, R. (2008). La production d'une identité autochtone en Côte d'Ivoire. Paris : Journal des anthropologues.

18. HOUDEINGAR, D. (2009). Les conflits d'usage entre principe de coexistence et principe de responsabilité. Paris: Programme scientifique (Idées).

19. IBO, G. (2006). Retraits de terres par les « jeunes » autochtones sur les anciens fronts pionniers de Côte d'Ivoire : expression d'une crise de transition intergénérationnelle. Montpellier : Colloque international "Les frontières de la question foncière". 
20. KAKULE, D. (2011). Résolution extra- judiciaire des conflits fonciers en territoire de masisi : procédure et valeur juridique. Mémoire de licence de droit. Université de Kisangani-RDC

21. KANA, P. (2017). Ventes illicites des terres et conflits intrafamiliaux dans la tribu Sian (RCI). Revue Africaine de Criminologie. Abidjan : EDUCI

22. KOUAME, G. (2006). Du conflit intrafamilial au conflit interethnique autour des transferts fonciers. Le cas de la société abouré (Basse Côte d'Ivoire). Montpellier: Colloque international "Les frontières de la question foncière".

23. LAVIGNE, D. (2002). Les pratiques populaires de recours à l'écrit dans les transactions foncières en Afrique rurale, Eclairages sur des dynamiques d'innovation institutionnelle. Document de travail de l'UR« Régulations Foncières, Politiques Publiques et Logiques d'Acteurs $"$.

24. LAWALI, S., MORMONT, M. et YAMBA, B. (2014). Gouvernance et stratégies locales de sécurisation foncière : étude de cas de la commune rurale de Tchadoua au Niger. Volume 14, numéro 1. Revue électronique en sciences de l'environnement.

25. LE ROY, E. (1996).La sécurisation foncière en Afrique (pour une gestion vraie des ressources renouvelables). Paris : Karthala.

26. MEILLASSOUX, C. (1964). Anthropologie économique des Gouro de Côte d'Ivoire : de l'économie de subsistance à l'agriculture commerciale. Paris : Mouton.

27. MERABET, O. (2006). Termes de Référence pour une étude sur le profil migratoire de la Côte d'Ivoire. Rapport final contrat cadre Europeaid: TRANSTEC.

28. MUMBERE, K. (2012). L'application de la loi dite foncière dans la résolution des conflits fonciers en territoire de Lubero en RDC. Graduat en droit privé. Université oficielle de Ruwenzori -

29. OUEDRAOGO, M. (2009). Problématique de l'accès à la terre et de la sécurisation foncière: cas des cultures maraîchères à Nouna. Mémoire de Géographie : Ecole Nationale d'Administration et de Magistrature (ENAM). Université de Burkina Faso.

30. OUMAROU, I. (2008). Femmes et développement local : analyse socio-anthropologique de l'organisation foncière au Niger : le cas de la région de Tillabery. Thèse pour l'obtention du Doctorat de sociologie. Université de Rennes 2. Haute-Bretagne.

31. RATIARAY, R. (1989). L'accès à la terre en droit rural malgache. Paris : Revue internationale de droit comparé. Volume 41 Numéro 3 pp. $637-707$ 
32. SOHINTO, D. (2001).Questions du genre liées aux conflits fonciers : Impact sur la production durable des produits vivriers du sud-Benin. Cotonou: Editions du Flamboyant

33. TALLET, B. et PARE, L. (1999). D'un espace ouvert à un espace saturé. Dynamiques foncières et démographique dans le département de Kouka (Burkina Faso). In Espace, populations, sociétés, Volume 17, Numéro 1 pp. 83-92

34. THAREAU, B. (2011). Réguler l'accès à la terre, la réinvention locale du corporatisme agricole. Paris : Cedex.

35. YODA, F. (2008).La sécurisation foncière en milieu rural au Burkina Faso. Programme spécifique "IDEES" - ERC (Conseil Européen de la Recherche) - Grant agreement for Advanced Investigator Grant (Sciences sociales).

36. ZONGO, M. (2009). Terre d'Etat, loi des ancêtres ? Les conflits fonciers et leurs procédures de règlement dans l'ouest du Burkina Faso. Paris : Journal des sciences sociales. 